\title{
Wireless Network Technology Course Reform
}

\author{
Luqiao Zhang *, Juan Wang and Zhe Ding \\ Department of Internet of Things, Chengdu University of Information Technology \\ "Corresponding author. Email: zhanglq@cuit.edu.cn
}

\begin{abstract}
Facing various challenges, including theoretic study of the details about network protocols easily bores students, students may have very different learning curves, and so on. To solve the above-mentioned problems, we apply the online and offline mixed teaching mode, flipped classroom, outcome-based evaluation to wireless network technology. Through We get very positive feedback from both students and survey conducted by undergraduate academic affairs office.
\end{abstract}

Keywords: Online and offline mixed teaching mode, Flipped classroom, Students centered and Outcomebased evaluation.

\section{INTRODUCTION}

The ubiquitous Wi-Fi network in offices, homes, cafes and shopping malls greatly facilitates Internet access. 4G smart phones allow us to maintain network connection even when we are on the highway. Therefore, it is necessary to include wireless network theoretical knowledge and practical ability in bachelor program for undergraduate students who major in computer science and engineer. Furthermore, when students finish the course, they should be able to solve real world wireless network engineering problem by what they have learned in the course.

Based on the above observation, we offered wireless network technology course as an extension of computer network course ever since 2013-2014 academic year. For now, it has been running for 7 consecutive academic years. In the past seven years, we have compiled our own textbook, wireless network technology - principle, security and practice, recorded the course video and published it on the online course platform, carried out the student-centered and outcome-based evaluation, and received very positive comments from students.

\section{COURSE OBJECTIVES}

When students complete the course, they should master not only the theories, but also have the ability to put those theories into practice. For example, configuring a Wi-Fi router, deploying a wireless network according certain user requirement, and so forth.
To be more specific, first of all, students should understand the basic concepts and history of wireless network technology, and the principle of wireless data transmission. Secondly, students should master the protocol details of 802.11, Bluetooth, cellular network and other typical wireless network technologies. Thirdly, on the basis of understanding the relevant protocols, they should be able to configure network devices, and to do packet analysis and network security test by using tools like Wireshark, Aircrack-ng and other tools. One step further, students should know how to use MATLAB to do network simulation and design a data transmission program on their own. Last but not least, they can combine theoretical and practical knowledge to solve practical problems such as enhance Wi-Fi network coverage in large houses and exploits vulnerability of a wireless router.

\section{CHALLENGES}

It is not an easy job to achieve those objectives mentioned in section 2. Based on students' feedbacks, our experiences and employers' survey, we believe that we are facing the following three challenges in the teaching process:

Firstly, the employer's demand for graduates requires that we have to emphasize the training of practical skills and enable students to use the knowledge to solve practical engineering problems.

Secondly, the very limited time in classroom and uniform course schedule are difficult to meet the 
individualized requirement of students with different learning curves.

At last, the traditional teacher-centered teaching method makes the study of theoretical knowledge very boring, and it is difficult to stimulate students' interest in learning and make them proactive rather than reactive.

\section{METHODS}

Generally speaking, the content of wireless network technology includes: theory, practice and the ability to solve real world engineering problem, which are shown in Figure 1. According to the learning curve of different contents, we have carried out the mixed teaching reform of online and offline.

In the study process, students need to complete preview before class (especially the practical link), review after class (especially the key and difficult points), homework and Q\&A after class by online method.

When we in classroom, we mainly analyze the key and difficult points, such as: Wi-Fi WPA2 security protocol, the key generation process under PSK mode, and the structure of encryption key. Of course, we will demonstrate the operation and explains those steps where students might make mistake. For example, Wireshark configuration file modification and obtaining administrator privilege. In order to monitor the students' study status, periodic quizzes are arranged. At the same time, students should also independently complete the experimental operation. Finally, it is also the most important thing, explore the solution for real world engineering problem through in classroom discussion, in which the performance weighs a lot in the final score.

Through the above instructional design, a lot of classroom time is saved, especially the detailed steps and playbacks for the experiment operation steps, which greatly reduces the time required for students to complete the experiment. In addition, the viewing time for online course video also fully takes into account in the final course grade. The students who learn at a very fast pace and those who are slow to get started can both be benefited from such scheme.

In general, the learning process is illustrated in Figure 2. The process begins with preview course video online, afterwards in classroom learning with quiz. Then, students complete the required experiment on their own in the lab. Followed discussion can be carried out. At last, the homework and the review.

In order to evaluation the learning outcome, different assessment methods are designed for different study activities and course content. For instance, the time spent in watching online course video in preview and review stage, the quiz grade, the lab report score, the performance in class discussion, and homework grade.

At the end of the course, by combining and summarizing the professors' experiences, students' feedbacks, plus the recent advances in wireless network technology, both the course content and instructional design are updated and improved.

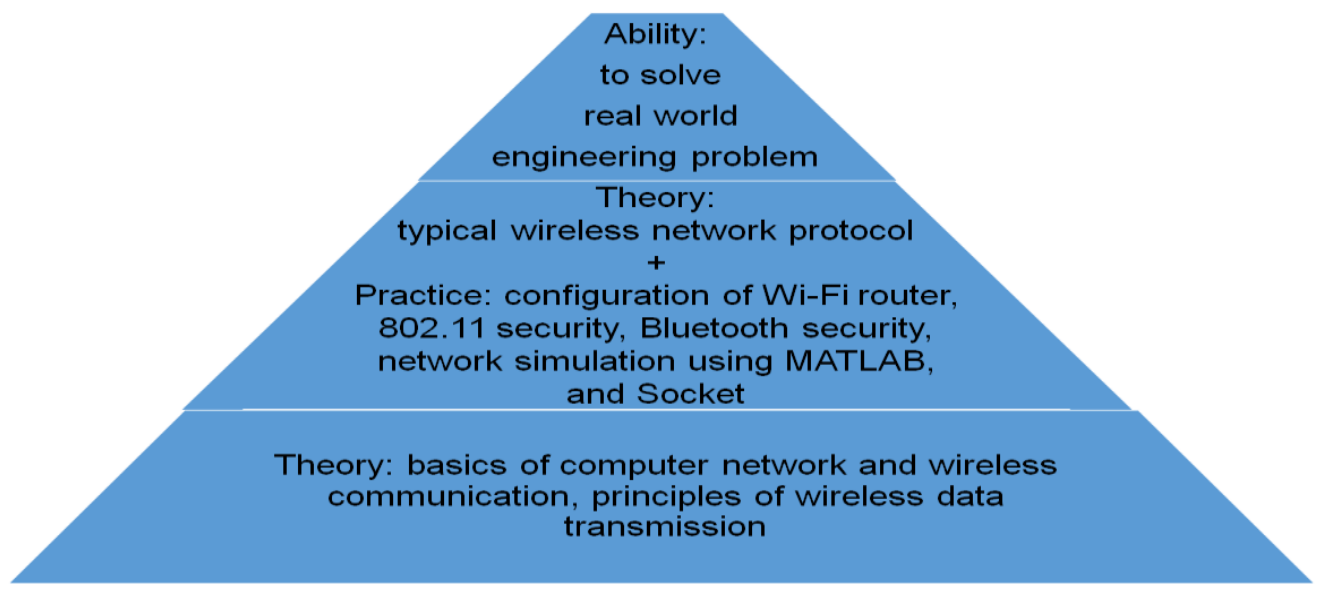

Figure 1. Course content pyramid

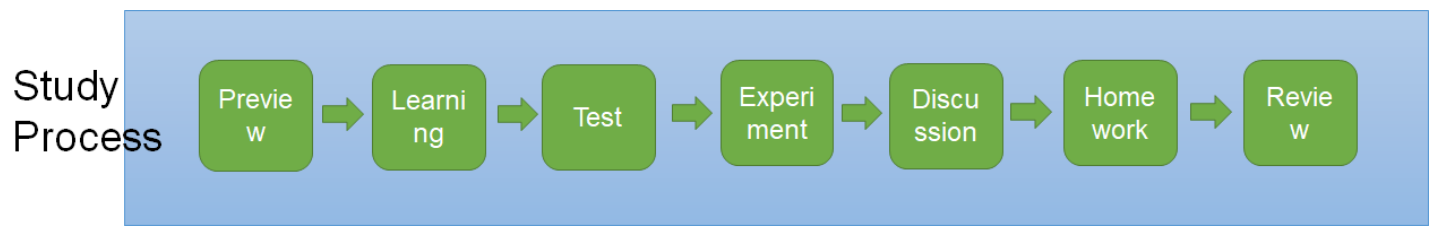

Figure 2. Study process 


\section{INSTRUCTIONAL DESIGN EXAMPLES}

We will further illustrate the course design by an example in this section. The teaching objective of this part is to solve the security problems in Wi-Fi network and enhance the system security.

First of all, through the phenomenon of 'wi-fi squatter' in real life, the concept is introduced. Then, the WEP, WPA, WPA2 security protocols and QSS security configuration mode are explored in detail. Then, the students are asked to use BEINI feeding bottle, Aircrack-ng and Wireshark security tools to crack the passphrase of a Wi-Fi network in WPA2-PSK mode, initialize a secure connection using QSS and crack the PIN of a Wi-Fi router with QSS function, capture and analyze wireless network packet by using Wireshark. After all that, we can discuss how to enhance the security of a Wi-Fi network. When the discussion ends, students' can do their homework and review according to their own situation.

\section{CONCLUSIONS}

Through the striving and continuous improvement of our team, the wireless network technology received very positive feedbacks from students, and the teaching evaluation results have been ranked in the top $10 \%$ in our university. As a result, professors have been awarded with no surprise. And we think following ideas and measures are what should be preserved in the future.

1) Under the guidance of the New Engineering Disciplines, the instructional design focuses on solving real world engineering problems, especially the application of flipped classroom to discussion. On one hand, it improves the students' learning initiative and enthusiasm. On the other hand, it really fulfills the curriculum goal of cultivating students' engineering ability and literacy.

2) In order to meet the individual study needs of different students, we have made great efforts in the construction of curriculum resources. We have compiled the teaching materials closely combined with theory and practice, collected and sorted out a large number of video resources, recorded the course videos by ourselves, and practiced the student-centered engineering teaching concept.

3) In order to test the students' learning output, according to the characteristics of different learning contents and learning links, the assessment link is designed to comprehensively evaluate the students' outcome. Through feedback and continuous improvement, a closed loop of improvement based on feedbacks is established.

\section{AUTHORS' CONTRIBUTIONS}

Luqiao Zhang conducts this project, Juan wang makes her contribution mainly in course content compile, and Zhe ding is in charge of online resources maintenance.

\section{ACKNOWLEDGMENTS}

This work is supported by Sichuan Educational and Teaching Reform Project (JG2018-525) and Chengdu University of Information Technology Educational and Teaching Reform Project (JY2018029)

\section{REFERENCES}

[1] Luqiao Zhang, Juan Wang, Fei Li and Lei Shi, The Implementation of the CDIO Initiative in CUIT, Proceedings of the 12th International CDIO Conference, Turku, Finland, June 12-16, 2016

[2] Liangbin Yang, Xinli Zhou, Sihan Liu, Zisen Sang, Jin Wang, Research on the Training Mechanism and Model of Network Space Security Personnel under the Background of Big Data, Intelligence Journal, 2016, 35(12):80-87

[3] Kui Meng, Gongshen Liu, Jianhua Li, Zhi Xue, Information Security Practice Course Construction Based on Innovative Education,

[4] Weiwei Hong, Research and Practice of SPOC Flip Classroom Teaching Based on Learning Passthrough, Journal of Higher Education 2020, 32:109-112

[5] Yun Liu, Zhengpeng, Di, Shimei Li, OBE-based "Three-Three Systems" Applied Talent Training Model, Journal of Higher Education 2020, 32: 173176 